\title{
EVOLUTION \\ DE LA QUANTITÉ D'OVULES RÉCOLTÉS ET CONSERVATION DE LEUR APTITUDE A ÊTRE FÉCONDÉS AU COURS DE LA PÉRIODE POST OVULATOIRE CHEZ LA TRUITE ARC EN CIEL
}

\author{
Anne-Marie ESCAFFrE, J. PETIT, R. BILLARD \\ I.N.R.A., Laboratoire de Physiologie des Poissons \\ 78350 JOUY-EN-JOSAS, France
}

\section{RESUME}

Une expérimentation visant à suivre pendant la saison de reproduction l'évolution de la quantité et de la qualité des ovules produits après ovulation a été conduite sur truite arc-en-ciel.

Dans une première expérience, des femelles ont été vidées du maximum de leurs ovules le jour de l'ovulation (JO) et aux dates suivantes $18, J 15, J 21$ et 
J30. $90 \%$ du nombre total d'ovules et $96 \%$ des ovules fécondables sont récoltés au cours des deux premiers prélèvements (JO et $\mathrm{J} 8$ ). Le pourcentage d'ovules œillés à JO est plus élevé chez les femelles ovulant précocement que chez ies femelles à ovulation tardive, suggérant, soit une plus forte rétention des ovules, soit l'apparition d'un assynchronisme de l'ovulation lorsque la période de ponte progresse.

Une deuxième expérience a consisté à laisser une partie des ovules dans la cavité générale après ovulation. Dans ces conditions, la fécondabilité des ovules se maintient pendant 30 jours pour des femelles de grande taille qui ont déjà assuré plusieurs reproductions. Au contraire, la fécondabilité chute après un séjour des ovules de 15 jours dans la cavité cœlomique de petites femelles à leur première ou deuxième saison de reproduction.

\section{CHANGES IN THE YIELD OF EGGS AND OF THEIR FERTILITY AT VARIOUS TIMES AFTER OVULATION IN THE RAINBOW TROUT}

\section{SUMMARY}

The quantity and quality of eggs released by rainbow trout when stripped at various times after ovulation were studied in two experiments.

Fish which had been fully stripped on the day of ovulation (JO) were stripped, $8,15,21$ and 30 days later $(18, J 15, J 21, J 30) .90 \%$ of the total number of eggs and $96 \%$ of the number fertilizable were harvested in the first two strippings $(\mathrm{JO}, \mathrm{J} 8)$. The percentage collected at $\mathrm{JO}$ was greater for early spawners suggesting either a greater retention of ovulated eggs or the appearance of asynchronous ovulation amongst late spawners.

When fish were incompletely stripped the retained eggs were fertile for 30 days in large fish which had already spawned several times whereas in small fish at first or second reproduction eggs lost their fertility after 15 days.

\section{INTRODUCTION}

L'absence de comportement reproducteur des géniteurs de truite arc-enciel en élevage implique la réalisation de l'insémination artificielle.

La collecte des gamètes et principalement des ovules est faite manuellement à un moment déterminé empiriquement par le pisciculteur. Le temps écoulé entre l'ovulation et la collecte est quelquefois assez long et la réussite de la fécondation peut être affectée par des phénomènes de vieillissement des ovules.

Dans la pratique, it est impossible de repérer le moment exact de l'ovulation, et il est intéressant d'essayer de déterminer après l'ovulation l'intervalle de temps pendant lequel l'éleveur pouvait espérer un taux de fécondation élevé. II existe déjà un certain nombre de données dans la littérature et pour NOMURA et al. (1974) et SAKAl et al. (1975) la fécondabilité des ovules de truite arc-en-ciel peut se maintenir pendant 10 jours, permettant l'obtention de plus de $70 \%$ d'œufs œillès.

Une partie de l'ètude suivante a déjà été présentée par ailleurs (ESCAFFRE et al., 1976). 


\section{MATERIEL ET METHODES}

Les expériences ont été menées sur la truite arc-en-ciel Salmo jairdneri Richardson. Les géniteurs en provenance de deux piscicultures ont été introduits au laboratoire avant leur maturité sexuelle. La température d'élevage a varié entre $10-12{ }^{\circ} \mathrm{C}$ pendant l'expérience.

Les femelles sont examinées quotidiennement afin de déterminer le.jour de l'ovulation (JO). L'insémination artificielle est pratiquée après dilution du sperme au $1 / 1000$ e avec le dilueur précédemment décrit (PETIT et al., 1973).

Deux séries d'expériences ont été réalisées :

1. Evolution de la production d'ovules et de leur fécondabilité au cours de la période post-ovulatoire (variations quantitative et qualitative dans la production d'ovules)

Chaque femelle est vidée du maximum de ses ovules en une seule fois (mode prélèvement I) le jour de l'ovulation (J0). Ce mode de prélèvement correspond à la méthode classiquement utilisée en pisciculture. A des temps croissants après le strippage $(15,21$ et 30 jours) les femelles sont repassées et les quantités maximum d'œufs sont à nouveau recueillies et mesurées. Le dernier prélèvement est effectué avant sacrifice de la femelle. Pour chacun des prélèvements les ovules sont fécondés; chaque prélèvement est fractionné en 3 parties qui sont chacune fécondées par un mâle différent. Les mâles utilisés pendant la durée de l'expérience ont été prélevés dans un groupe de 15 individus isolès en début d'expérience où ils étaient alors en début de spermiation. Les femelles de l'expérience I étaient exclusivement constituées de jeunes individus âgés de 3 ans et effectuant leur première ou deuxième reproduction.

\section{après ovulation}

2. Evolution de la fécondabilité des ovules laissés dans la cavité générale

Dans cette expérience une partie seulement des ovules disponibles est prélevée aux temps suivants : JO, J8, J15, J21, J30 (mode de prélèvement II). A $J 30$ la totalité des ovules restants est recueillie avant sacrifice de la femelle. La fécondation est pratiquée comme en I. Deux populations différentes de femelles ont été utilisées. Les unes sont de petite taille $(500$ à $700 \mathrm{~g})$ et âgées de 3 ans, les autres sont âgées de plus de 4 ans et pèsent de 1,5 à $2 \mathrm{~kg}$. Dans ce cas les ovules recueillis sont supposés avoir vieilli dans la cavité générale pendant des durées correspondant aux dates de prélèvement, à moins qu'un assynchronisme de l'ovulation n'ait entrainé une hétérogénéité des lots d'ovules récoltés.

\section{RESULTATS}

Expérience I : Evolution quantitative et qualitative de la production d'ovules au cours de la période post-ovulatoire dans le cas de la récolte du maximum d'ovules à chaque récolte

L'entrée en ovulation des différentes femelles de l'expérience s'est étalèe sur 75 jours. Les informations recueillies sur l'évolution de la production d'ovules et leur fécondabilité sont les suivantes :

1. Valeurs moyennes recueillies pendant la période post-ovulatoire :

La production maximale d'ovules est obtenue le jour de l'ovulation (JO) (près de 1500 ovules, fig. 1). Au prélèvement suivant, à 8 jours, la récolte est significativement plus faible $(P<0,001)$. Au-delà de 18 , les quantités recueillies sont négligeables. 
La fécondabilité des ovules est maximale à JO et décroit ensuite significativement $(P<0,01$ ) (tableau $I$ )). La variation individuelle du taux de fécondation des ovules exprimée par le coefficient de variation augmente avec le temps écoulé depuis l'ovulation (fig. 2).

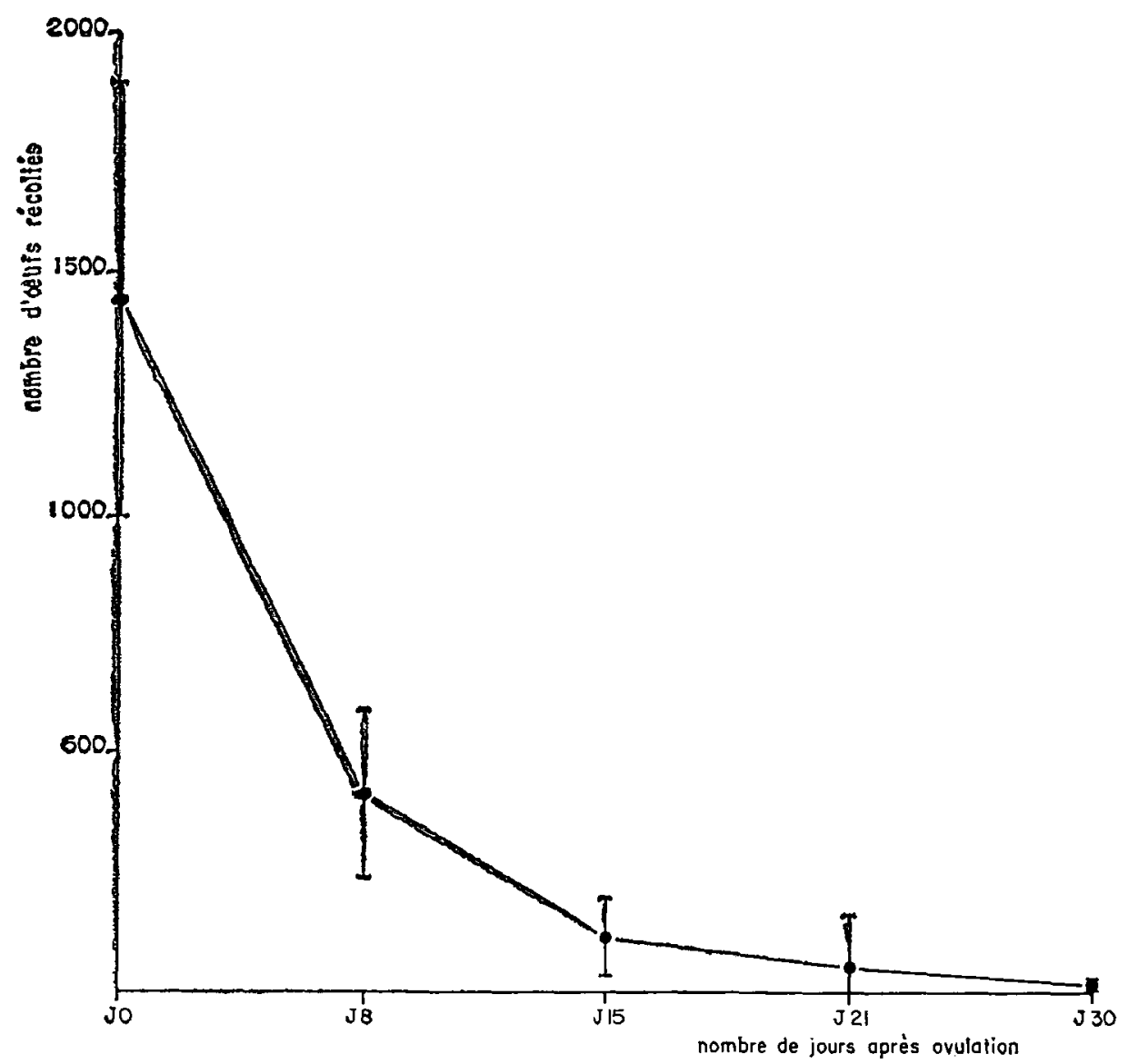

Figure 1 - Quantité d'œufs récoltés en fonction du nombre de jours après ovulation. Prélèvement I.

TABLEAU 1

RESULTATS DU PRELEVEMENT I

\begin{tabular}{|c|c|c|c|c|}
\hline $\begin{array}{c}\text { jour de } \\
\text { prélèvement }\end{array}$ & $\begin{array}{l}\% \text { d'œufs } \\
\text { récoltés }\end{array}$ & $\begin{array}{c}\% \text { de } \\
\text { fécondation de } \\
\text { chaque collecte }\end{array}$ & $\begin{array}{l}\% \text { d'œufs } \\
\text { fécondés } \\
\text { récoltés }\end{array}$ & $\begin{array}{c}\text { Nombre } \\
\text { de } \\
\text { femelles }\end{array}$ \\
\hline 0 (ovulation) & 72,65 & 85,28 & 77,71 & 8 \\
\hline 8 & 20,70 & 71,65 & 96,32 & 8 \\
\hline 15 & 5,04 & 49,38 & 99,44 & 7 \\
\hline 21 & 1,07 & 41,76 & 100 & 6 \\
\hline 30 & 0,54 & 0 & - & 6 \\
\hline
\end{tabular}




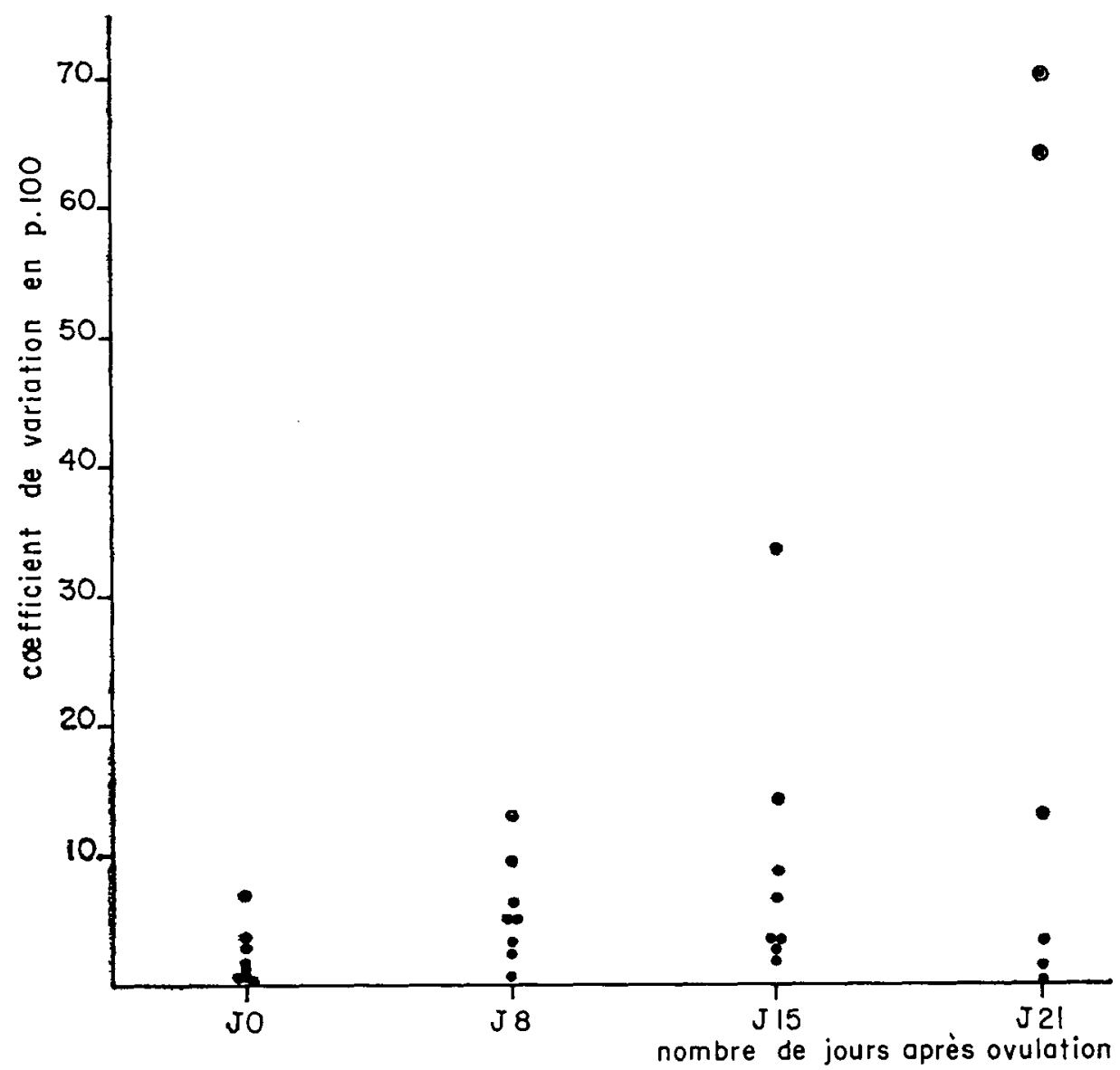

Figure 2 - Variabilité du taux de fécondation en fonction du temps après ovulation. Prélèvement $\mathbf{I}$.

En conclusion, plus de $90 \%$ des ovules sont récoltés dans les 8 jours qui suivent l'ovulation (ponte totale + une $\propto$ repasse $»$ ). Si le pourcentage est exprimé par rapport aux ovules fécondables (les seuls intéressants) la quasi totalité $(96 \%)$ de ces derniers est aussi recueillie durant ces 8 jours postovulation.

2. Evolution du nombre et de la fécondabilité d'ovules récoltés à JO en fonction du mois où l'ovulation a eu lieu.

Si le nombre d'ovules récoltés à JO est représenté en fonction du mois où l'ovulation a été observée, on constate (fig. 3) que le rendement de la collecte décroit fortement lorsque la saison de ponte avance. L'évolution du taux de fécondation de ces ovules prélevés à JO est plus stable (fig. 4). Les ovulations précoces et tardives présentent des taux de fécondation plus faibles, mais le facteur sperme doit être pris en considération ici.

Expérience II : Evolution de la fécondabilité des ovules laissés dans la cavité générale après ovulation (fig. 5)

Pour toutes les femelles mises en expérience, la fécondabilité des ovules se maintient au-dessus de $80 \%$ pendant les 15 jours suivant l'ovulation. La fécondabilité évolue ensuite différemment suivant la taille des femelles; elle 


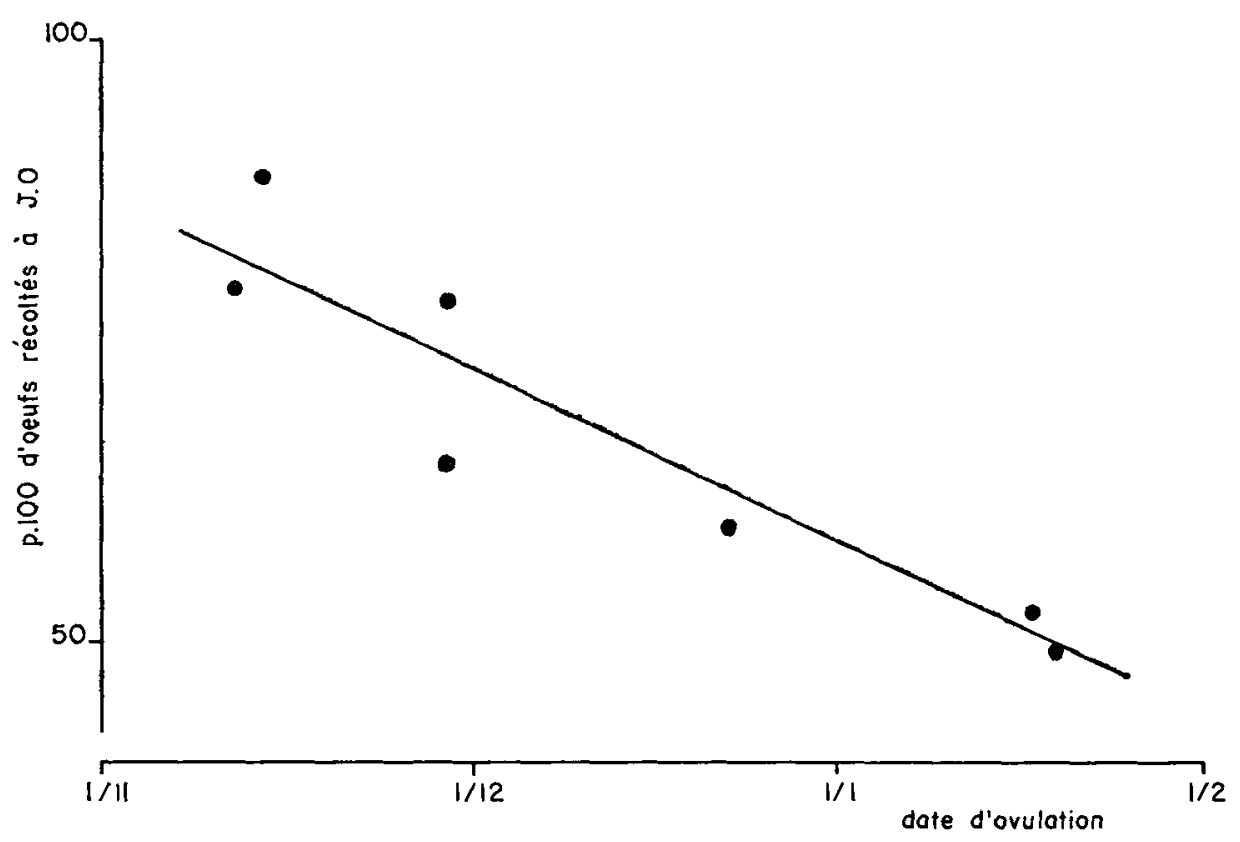

Figure 3 - Nombre d'œufs récoltés le jour de l'ovulation en fonction de la date d'ovulation. Prélèvement I.

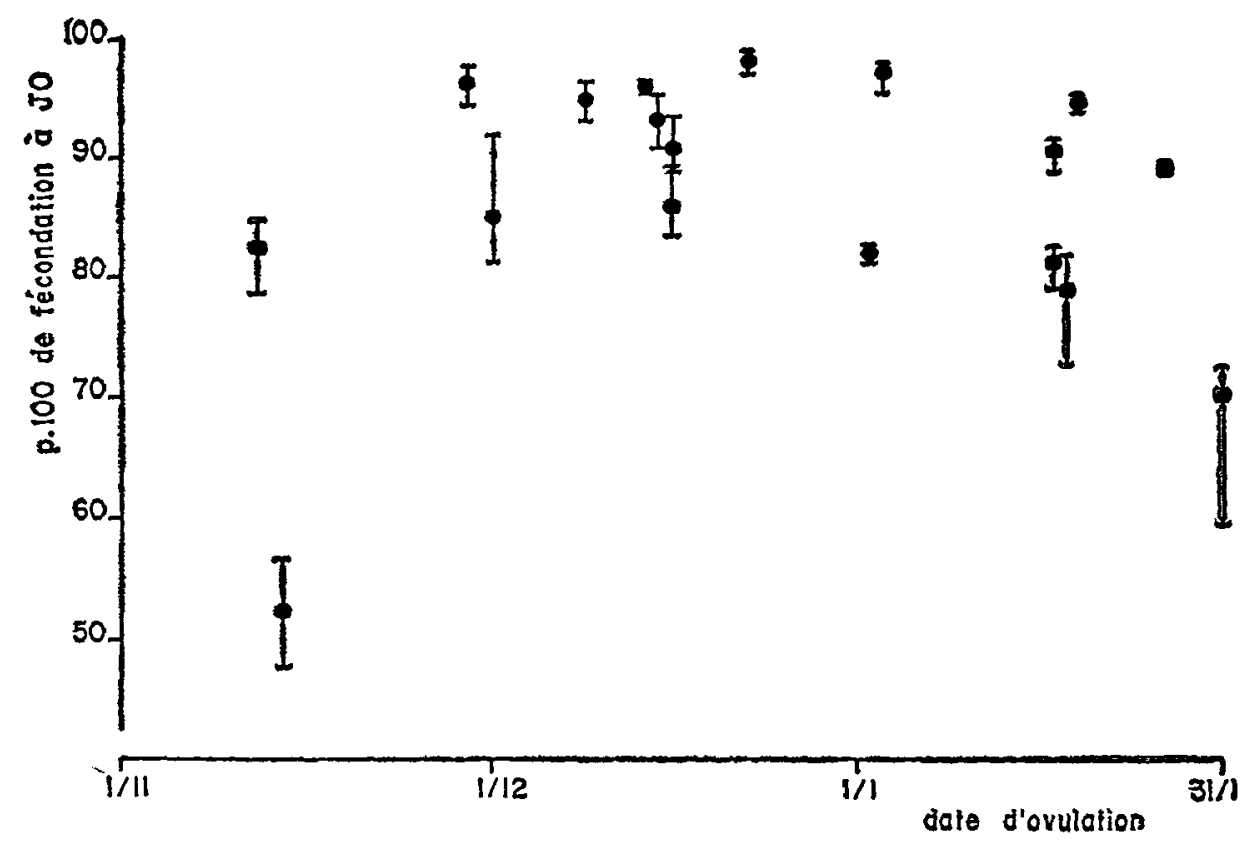

Figure 4 - Taux de fécondation à l'ovulation en fonction de la date d'ovulation. 


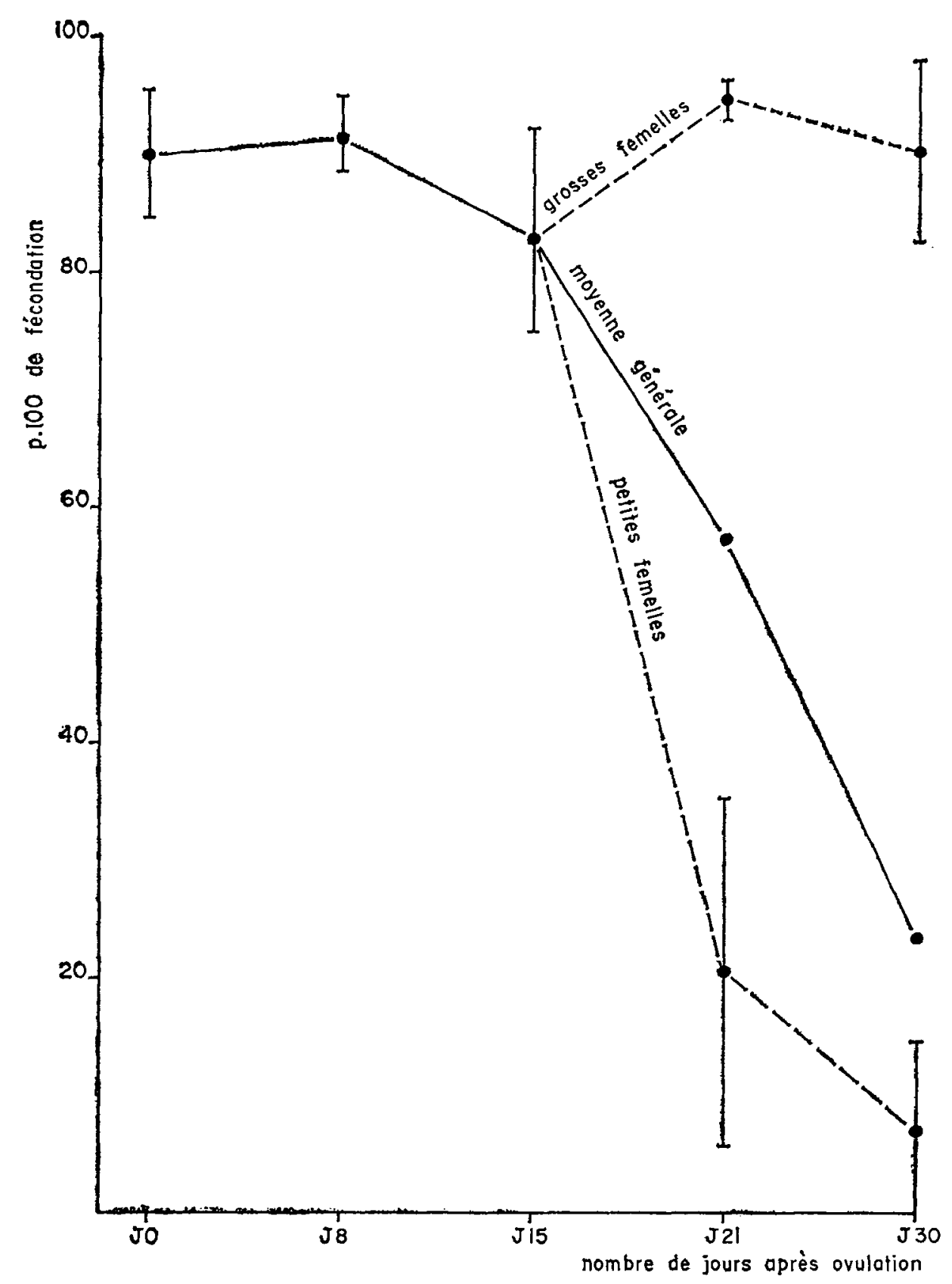

Figure 5 - Pourcentage de fécondation en fonction du nombre de jours après ovulation. Prélèvement II. 
décroit rapidement après 15 jours pour les petites femelles et se maintient à plus de $80 \%$ pendant les 30 jours suivant l'ovulation pour les femelles de forte taille.

\section{DISCUSSION ET CONCLUSION}

La présente étude montre que des ovules peuvent ètre laissés dans la cavité générale de truite arc-en-ciel femelles sans perte de leur fécondabilité, pendant des durées relativement longues ( 30 jours); mais cette aptitude semble dépendre de la qualité des femelles (taille dans le cas présent). II est donc vraisemblable que l'histoire des géniteurs soit un facteur déterminant (condition d'élevage, taille, conditions sanitaires, etc.). L'appréciation de cet intervalle de temps pendant lequel les ovules peuvent séjourner sans dommage dans la cavité générale après ovulation reste donc subjective. Dans la pratique piscicole, il semble qu'une rétention des ovules supérieure à une semaine soit à déconseiller en attendant de disposer de critères d'appréciation de la qualité de la femelle facilement identifiables.

D'après les données de la littérature, la rétention des œufs chez la truite arc-en-ciel se traduit par des modifications morphologiques importantes (NOMURA et al., 1974). Cette rétention peut aussi affecter l'état physiologique des femelles (TAKASHIMA et al., 1975); SAKAl et al. (1975) ont observé une chute de fertilité au-delà du 10 e jour, ce qui correspond aux valeurs observées sur les femelles de petite taille. Compte tenu des résultats d'expériences complémentaires portant sur la survie embryonnaire et post-embryonnaire, ces mêmes auteurs (SAKAl et al., 1975) conseillent de prélever les ovules dans les 5 à 7 jours suivant l'ovulation. L'aptitude des ovules à être fécondés diminue plus rapidement dans l'expérience I (Tabl. I) que dans l'expérience II - fig. 5 (comparaison sur les femelles de petite taille). Dans le premier cas, la quantité d'ovules laissés dans la cavité générale est beaucoup plus faible que dans le second. II se pourrait alors que la conservation soit moins bonne lorsque la masse d'ovules restants est réduite.

La diminution du rendement des collectes à JO pour les ovulations tardives (fig. 3) appelle une discussion. II peut s'agir d'une plus forte rétention des ovules dans le cas des ovulations tardives ou d'un assynchronisme dans l'ovulation (ovulations étalées sur plusieurs jours). ISLAM et al. (1973) qui ont étudié chez la truite arc-en-ciel les variations des pontes de reproducteurs femelles précoces et tardifs n'ont pas signalé un tel phénomène, mais ont montré que le nombre et le poids des ovules étaient plus élevés chez les femelles tardives. L'évolution des pourcentages de fécondabilité observés au cours de l'ensemble de la période de ponte (fig. 4) ne permet pas de conclure à une diminution de la fécondabilité des ovules en fin de période de reproduction car le facteur qualité du sperme n'a pu être apprécié, d'autant plus que ce dernier est susceptible d'évoluer au cours de la période de spermiation (CHEMAYEL, 1975 ; BILLARD et BRETON, 1976).

\section{En conclusion :}

1) La totalité des ovules de bonne qualité peut être recueillie en deux récoltes, l'une après l'ovulation et l'autre une semaine plus tard;

2) La survie des ovules laissés dans la cavité générale de la femelle après ovulation peut se maintenir pendant 30 jours, mais cela semble dépendre de facteurs liés à la femelle, facteurs qu'il n'est pas encore possible d'identifier objectivement. 


\section{REMERCIEMENTS}

Ce travail a été réalisé dans le cadre d'un contrat I.N.R.A.-C.S.P. II a été présenté au Congrès Européen des Ichtyologistes (Paris, sept. 1976).

\section{BIBLIOGRAPHIE}

BILLARD R., BRETON B., 1976. Sur quelques problèmes de physiologie du sperme chez les poissons Téléostéens. Ile Congrès Europe. Ichtyol., Paris, septembre 1976.

CHEMAYEL M., 1975. Etude de la variabilité du pouvoir fécondant du sperme en relation avec ses caractéristiques chez la truite arc-en-ciel (Salmo gairdneri Richardson). Thèse 3 e Cycle, Univ. Paris VI.

ESCAFFRE A.M., PETIT 1., BILLARD R., 1976. Evolution de la fécondabilité des ovules de truite arc-en-ciel laissés dans la cavité cœlomique après ovulation Ile Congrès Europe. Ichthyol., Paris, Septembre 1976.

ISLAM H., NOSE Y., YASUDA F., 1973. Eggs characteristics and spawning season of rainbow trout. Bull. Jap. Soc. Sci. Fish, 39, 741-751.

NOMURA M., SAKAI K., TAKASHIMA F., 1974. The over-ripening phenomenon of rainbow trout. I - Temporal morphological changes of eggs retained in the body cavity after ovulation. Bull. Jap. Soc. Sci. Fish, 40, 977-984.

PETIT J., JALABERT B.. CHEVASSUS B., BILLARD R., 1973. L'insémination artificielle de la truite. I - Effets du taux de dilution, du $\mathrm{pH}$ et de la pression osmotique du dilueur sur la fécondation. Ann. Hydrobiol., 4, 201-210.

SAKAI K., NOMURA M., TAKASHIMA F., OTO $H_{\text {., }}$ 1975. The over-ripening phenomenon of rainbow trout. II - Changes in the percentage of eyed eggs, hatching rate and incidence of abnormal alevins during the process of overripening. Bull. Jap. Soc. Sci. Fish, 41, 855-860.

TAKASHIMA F., NOMURA M., SAKAI K., 1975. The over-ripening phenomenon of rainbow trout. III - Histological studies on the physiological condition of over-ripened female. Bull. Jap. Soc. Sci. Fish., 41, 861-867. 